\title{
Modeling Lotic Organism Populations with Partial Differential Equations
}

\author{
Chase Viss* \\ Faculty Advisors: Thomas J. Clark ${ }^{\dagger}$ and Eric Eager ${ }^{\ddagger}$
}

January 18, 2016

\begin{abstract}
We present in this paper a mathematical model for a population of caddisfly larvae in the Upper Mississippi River, which can either live in the current of the water or fix themselves to large wood debris submerged throughout the river. The model consists of a system of partial differential equations which captures these coupled dynamics. After introducing the model, we give a qualitative analysis of the dynamics, which includes a steady state solution followed by a numerical solution to the system using a finite difference scheme implemented in R. Finally, we extend the model to a competitive system with the goal of capturing the dynamics of the interaction between native caddisfly larvae and invasive zebra mussels. Our results demonstrate that although analyzing the exact behavior of lotic organism populations remains a difficult task, utilizing mathematical models such as the one presented in this paper can lead to further knowledge regarding which characteristics of lotic organisms have greatest influence over population growth.
\end{abstract}

Keywords: mathematical biology, transport equation, finite difference method.

\section{Introduction}

Mathematical models are useful tools for describing, understanding, and predicting the behavior of biological systems - for example, the predator-prey model and the von Bertalanffy growth model [de Vries, 2006]. This paper introduces a mathematical model that describes the population of one or multiple lotic organisms whose growth depends on the presence of large wood debris (LWD), submerged or partially submerged pieces of wood, scattered throughout a river, here assumed to be the Upper Mississippi River. Our model is a system of partial differential equations that accounts for both river dynamics and biological growth of the organisms. After describing the development of the model, we perform a qualitative analysis of the system consisting of computer simulation of the ecosystem using a finite difference method as well as steady state prediction through analysis.

\footnotetext{
*e-mail: charles.viss@ucdenver.edu

${ }^{\dagger}$ e-mail: tom.clark@dordt.edu

${ }^{\ddagger}$ e-mail: eeager@uwlax.edu
} 


\section{Biological Background}

The populations of lotic organisms, creatures that inhabit fast-moving fresh water, are difficult to analyze. Due to the fact that they are in constant motion, it is nearly impossible to repeatedly or accurately measure their numbers. However, in 1981, Michael J. Wiley took a novel approach to this area of research by modeling the distribution of lotic chironomid larvae using differential equations in order to describe sediment preferences [Wiley, 1981]. Nevertheless, the precise behavior of many lotic populations remains largely unknown and is an ongoing area of research.

Here, a similar mathematical model will be presented to describe the behavior of caddisfly larvae, a species of lotic organisms that is abundant in areas such as the Upper Mississippi River (UMR). Caddisfly larvae play a significant role in processing large wood debris in the UMR, and in turn, have become an important food source for fish in the river and act as an integral part of the area's ecosystem. However, recent human activity such as deforestation and the removal of snags from the riverbed has drastically reduced the amount of large wood debris in the UMR, which has the potential to substantially affect organism population within the river. The model laid out in this paper serves to illustrate the relationship between caddisfly larvae population and large wood debris in the river. Numerical simulations based on our model demonstrate that the removal of large wood debris has adverse effects on organism population growth.

Additionally, the presence of the invasive zebra mussel in the UMR also has the potential to affect caddisfly larva population. Zebra mussels began to receive national attention in the United States during the late 1980's, when their densities reached over $750,000 / \mathrm{m}^{2}$ in certain areas of the Great Lakes [Schloesser/Naleba/Mackie, 1996]. This prolific organism is now abundant in many freshwater systems of North America, such as the Mississippi River, where it competes with caddisfly larvae by latching onto large wood debris and, thereby, reducing the surface area of large wood debris that is available for colonization. By extending our single-organism model to a multi-organism model, we can capture the dynamics of this interaction and demonstrate its overall impact on caddisfly larva population.

\section{Single-Organism Model}

The purpose of any mathematical model is to describe a physical system using mathematical concepts and definitions. In this case, a system of differential equations is used to describe a population of organisms in the Upper Mississippi River. As with any mathematical model, certain underlying assumptions guide the development of the model. First, the model treats the river as a one-dimensional system in space. At each point in the river is a certain quantity (typically measured in biomass) of caddisfly larvae. Also assumed to be located at each point in the river is some source of large wood debris to which the organisms may latch on and temporarily exit the river's current. Letting $C(x, t)$ denote the mass of caddisflies (in dry weight) in the current of the river at position $x$ and time $t$ and letting $N(x, t)$ denote the mass of caddisflies that are latched onto large wood debris, we wish to describe the behavior of $C$ and $N$ using a coupled system of differential equations. This system will be based on the following qualitative set of equations: 


$$
\begin{aligned}
& \frac{\partial C}{\partial t}=t r-l a+l e \\
& \frac{\partial N}{\partial t}=l a-l e+f
\end{aligned}
$$

where $t r$ is the transport of the caddisflies downstream, $l a$ is the rate at which caddisflies leave the river to latch onto the LWD, le is the rate at which caddisflies leave the LWD to reenter the river, and $f$ is the growth rate of caddisflies while on the LWD.

The transport of $C$ down the river requires us to consider the spatial derivative of $C$ in addition to the time derivative. This results in components of the well-known partial differential equation (PDE) called the transport equation. This equation is widely used to describe the horizontal advection of a substance throughout a domain over time - for example, the drift of a pollutant down a river. In this model, the transport equation enforces the current of the river, which transports the organisms downstream. Assuming a constant rate of advection, $v$, from the river we derive

$$
t r=-v \frac{\partial C}{\partial x}
$$

Note that the dimension of $v$ is distance/time. This equation states that the rate at which $C$ changes due to transport with respect to time is directly proportional to the rate at which $C$ changes with respect to location. In other words, as time increases by $\Delta t$ units, organisms located at an arbitrary location $x$ are moved to location $x+v(\Delta t)$ by the current of the river. The overall shape of the population does not change due to transport, but rather, it is translated downstream over time.

At each location $x$, a portion of the organisms in the current of the river latches on to LWD, where they may consume nutrients and increase in biomass. The rate at which this "latching" occurs, which is denoted la above, and has dimension mass/time, is determined by the location's current values of both $C$ and $N$ :

$$
l a=\frac{\alpha C}{1+h N^{2}}
$$

The parameter $\alpha$ has dimension 1/time and corresponds to the percentage of organisms in the river that would naturally attempt to latch on to vacant LWD. Additionally, the parameter $h$, whose dimension is $1 /$ mass $^{2}$, enforces the effect of overcrowding: as $N$ increases, less surface area is available on the LWD for colonization, reducing the rate at which organisms from the current will be able to latch on.

The rate at which organisms leave LWD and reenter the river, denoted le with dimension mass/time, at any given time and location is determined entirely by the size of the population $N$ latched onto the LWD:

$$
l e=\gamma N+\eta N^{2}
$$


As $N$ increases, the LWD becomes crowded and both $\gamma$ and $\eta$ increase the concentration of organisms that reenter the river. The parameter $\gamma$ has dimension $1 /$ time, where $1 / \gamma$ is the average amount of time spent on the log. The parameter $\eta$ has dimension $1 /$ (time $\cdot$ mass $)$. The linear term describes the portion of organisms that naturally release from the LWD to reenter the current, and the quadratic term describes those organisms that are forced to leave due to overcrowding.

Finally, the growth rate of organisms latched onto LWD, denoted $f$ with dimension mass/time, is described by the logistic growth equation:

$$
f=r N\left(1-\frac{N}{K}\right)
$$

The parameter $r$, whose dimension is $1 /$ time, represents the default rate at which the organisms grow. Once latched on to LWD, in addition to feeding off the organic matter of the wood, caddisfly larvae have the ability to feed off microorganisms in the current of the river using a built-in, filter-feeding apparatus [Haro, 2014]; the parameter $r$ takes both of these dynamics into account. Also, $K$, whose dimension is mass, represents the carrying capacity of the LWD, causing the rate at which organisms grow to approach zero as the population size approaches the $K$.

The terms $l a$, le, and $f$ are necessarily nonlinear in $N$ and/or $C$ to model competition for space in ecological systems, specifically systems like this one [Wiley, 1981].

The complete system of partial differential equations for this model-equations (5) and (6) - can now be constructed by combining growth rates (1)-(4). First, $\frac{\partial C}{\partial t}$ is formed by combining the transport equation with $l a$ and $l e$ :

$$
\begin{aligned}
\frac{\partial C}{\partial t} & =t r-l a+l e \\
& =-v \frac{\partial C}{\partial x}-\frac{\alpha C}{1+h N^{2}}+\left(\gamma N+\eta N^{2}\right)
\end{aligned}
$$

Similarly, $\frac{\partial N}{\partial t}$ is formed by combining $l a$ and le with $f$ :

$$
\begin{aligned}
\frac{\partial N}{\partial t} & =l a-l e+f \\
& =\frac{\alpha C}{1+h N^{2}}-\left(\gamma N+\eta N^{2}\right)+r N\left(1-\frac{N}{K}\right)
\end{aligned}
$$

where $v, \alpha, h, \gamma, \eta, r$, and $K$ are positive parameters. With these two equations, the model is completed by specifying an initial condition $C(x, 0)$ for the current of the river, an initial condition $N(x, 0)$ for the LWD in the river, and a boundary condition $C(0, t)$ for the river, which specifies the quantity of organisms that enter the system at any given time. 


\section{Non-Dimensionalization}

A useful technique for analyzing any mathematical model is the process of non-dimensionalization: substituting all components of the model with dimensionless counterparts. Advantages of using this technique include reducing the number of parameters that appear in the equations, discovering how certain variables and parameters are related to each other, and determining which variables and parameters are most relevant to the model.

The first step of this process is to determine the dimensions of all variables and parameters of the model. Let $M$ denote the dimension mass, $D$ denote distance, and $T$ denote time. Then variables $C$ and $N$ (along with the parameter $K$ ) have dimension $M$, variable $x$ has dimension $D$, and variable $t$ has dimension $T$. Furthermore, parameters $\alpha, \gamma, \eta$, and $r$ all have dimension $T^{-1} ; v$ has dimension $D T^{-1} ; h$ has dimension $M^{-2}$; and $\eta$ has dimension $M^{-1} T^{-1}$.

Once the dimensions of all variables and parameters have been realized, new dimensionless variables can be created by multiplying or dividing each current variable by one or multiple parameters such that the dimension of the resulting combination is $M^{0} D^{0} T^{0}$. In this model, dimensionless variables $\bar{C}, \bar{N}, \bar{t}$, and $\bar{x}$ can be formed by the following combinations:

$$
\begin{aligned}
\bar{C} & =\frac{C}{K} \\
\bar{N} & =\frac{N}{K} \\
\bar{t} & =r t \\
\bar{x} & =\frac{r}{v} x
\end{aligned}
$$

Next, each partial derivative of these new variables can be calculated by using the chain rule. For example:

$$
\frac{\partial \bar{C}}{\partial \bar{t}}=\frac{\partial\left(\frac{C}{K}\right)}{\partial \bar{t}}=\frac{1}{K} \frac{\partial C}{\partial \bar{t}}=\frac{1}{K} \frac{\partial C}{\partial t} \frac{\partial t}{\partial \bar{t}}=\frac{1}{K} \frac{\partial C}{\partial t}\left(\frac{1}{r}\right)=\frac{1}{K r} \frac{\partial C}{\partial t}
$$

Similarly, it can be shown:

$$
\begin{gathered}
\frac{\partial \bar{N}}{\partial \bar{t}}=\frac{1}{K r} \frac{\partial N}{\partial t} \\
\frac{\partial \bar{C}}{\partial \bar{x}}=\frac{v}{K r} \frac{\partial C}{\partial x}
\end{gathered}
$$

Substituting (5) and (9) into (7) yields: 


$$
\begin{aligned}
\frac{\partial \bar{C}}{\partial \bar{t}} & =\frac{1}{K r}\left(-v \frac{\partial C}{\partial x}-\frac{\alpha C}{1+h N^{2}}+\left(\gamma N+\eta N^{2}\right)\right) \\
& =\frac{-v}{K r}\left(\frac{K r}{v} \frac{\partial \bar{C}}{\partial \bar{x}}\right)-\frac{\frac{\alpha}{K r} C}{1+h N^{2}}+\left(\frac{\gamma}{K r} N+\frac{\eta}{K r} N^{2}\right) \\
& =-\frac{\partial \bar{C}}{\partial \bar{x}}-\frac{\frac{\alpha}{r} \bar{C}}{1+h K^{2} \bar{N}^{2}}+\left(\frac{\gamma}{r} \bar{N}+\frac{\eta K}{r} \bar{N}^{2}\right) \\
& =-\frac{\partial \bar{C}}{\partial \bar{x}}-\frac{\pi_{1} \bar{C}}{1+\pi_{2} \bar{N}^{2}}+\left(\pi_{3} \bar{N}+\pi_{4} \bar{N}^{2}\right)
\end{aligned}
$$

where $\pi_{1}=\frac{\alpha}{r}, \pi_{2}=h K^{2}, \pi_{3}=\frac{\gamma}{r}$, and $\pi_{4}=\frac{\eta K}{r}$ are dimensionless parameters. Similarly, substituting (6) into (8) results in:

$$
\frac{\partial \bar{N}}{\partial \bar{t}}=\frac{\pi_{1} \bar{C}}{1+\pi_{2} \bar{N}^{2}}-\left(\pi_{3} \bar{N}+\pi_{4} \bar{N}^{2}\right)+\bar{N}(1-\bar{N})
$$

Equations (10) and (11) together act as the dimensionless counterpart to the original system. Note how both equations include only four parameters compared to the seven parameters shared by the equations of the original system. This dimensionless system may be a helpful guide in determining which parameters are of most interest to the model.

\section{$5 \quad$ Finding a Steady State Solution}

The first question to be addressed by this model is whether or not the population of organisms on the LWD and in the river will eventually reach an equilibrium. Mathematically, this means we are interested in determining whether or not the system has a steady state solution. In this system, a steady state occurs when both $\frac{\partial C}{\partial t}$ and $\frac{\partial N}{\partial t}$ equal 0 :

$$
\begin{aligned}
& 0=\frac{\partial C}{\partial t}=-v \frac{\partial C}{\partial x}-l a+l e \\
& 0=\frac{\partial N}{\partial t}=l a-l e+f
\end{aligned}
$$

Summing and simplifying these two equations yields the equality:

$$
f=v \frac{\partial C}{\partial x}
$$

Therefore, an equilibrium occurs in this system when, at all locations, the growth rate of the organisms on the LWD is equal to the rate at which organisms leave that location due to the current of the river.

Furthermore, it is possible to estimate functions $C_{e q}(x)$ and $N_{e q}(x)$ that describe the population's sizes when this equilibrium occurs. First, by setting $\frac{\partial N}{\partial t}$ equal to 0 and solving for $C$, we obtain an algebraic equation for $C$ entirely in terms of $N$ : 


$$
C=\frac{1+h N^{2}}{\alpha}\left[\gamma N+\eta N^{2}-r N\left(1-\frac{N}{K}\right)\right]
$$

Differentiating each side of (13) with respect to $x$ yields an equation for $\frac{\partial C}{\partial x}$ :

$$
\frac{\partial C}{\partial x}=\left[4\left(\frac{h \eta}{\alpha}+\frac{r h}{\alpha K}\right) N^{3}+3\left(\frac{h \gamma-r h}{\alpha}\right) N^{2}+2\left(\frac{\eta}{\alpha}+\frac{r}{\alpha K}\right) N+\frac{\gamma-r}{\alpha}\right] \frac{\partial N}{\partial x}
$$

Next, manipulating (12) results in another equation for $\frac{\partial C}{\partial x}$ :

$$
\frac{\partial C}{\partial x}=\frac{r}{v} N\left(1-\frac{N}{K}\right)
$$

Finally, equating (14) and (15) and then solving for $\frac{\partial N}{\partial x}$ yields an equation for $\frac{\partial N}{\partial x}$ entirely in terms of $N$ :

$$
\frac{\partial N}{\partial x}=\frac{\frac{r}{v} N-\frac{r}{K v} N^{2}}{4\left(\frac{h \eta}{\alpha}+\frac{r h}{\alpha K}\right) N^{3}+3\left(\frac{h \gamma-r h}{\alpha}\right) N^{2}+2\left(\frac{\eta}{\alpha}+\frac{r}{\alpha K}\right) N+\frac{\gamma-r}{\alpha}}
$$

\section{Predicted Equilibrium}

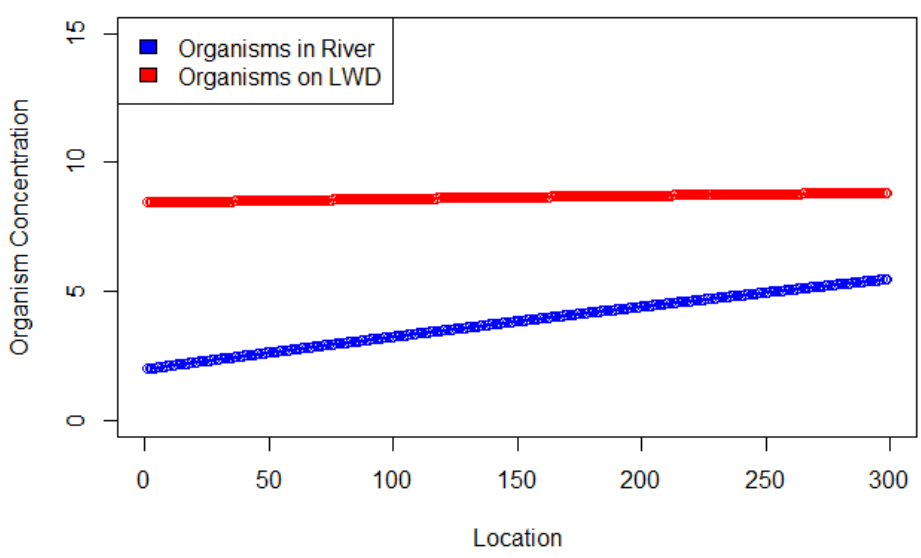

Figure 1: Predicted equilibrium values using $N_{e q}(0)$

Treating (16) as an ordinary differential equation, it is now possible to use standard numerical methods for solving ODE's (such as the Runge-Kutta method) to estimate $N_{e q}(x)$ at the system's steady state (given an initial value $N_{e q}(0)$ ). To obtain this initial value, equation (13) along with $C(0, t)=C_{e q}(0)$ (assuming that it is a known boundary condition constant) can be used to solve for $N_{e q}(0)$. Once values for $N_{e q}(x)$ are estimated, equation (13) can again be used to calculate $C_{e q}(x)$ at each appropriate location. Figures 1 and 2 illustrate how a numerical solution of the model (described in the following section) results in values for $C$ and $N$ that approach this predicted equilibrium. 


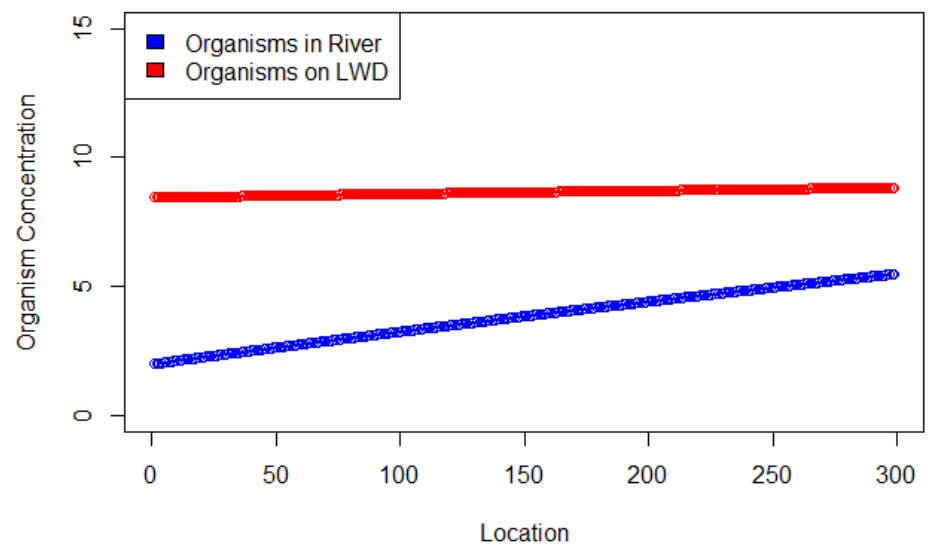

Figure 2: Results of simulation using Finite Difference scheme

\section{A Numerical Solution using Finite Differences}

Due to the complexity of the partial differential equations of this system, an analytic solution for this model is difficult to achieve. However, by performing a discretization of the system and using finite differences, a computer can be used to calculate an approximate solution numerically.

The motivation behind any finite difference method is the following approximation of a derivative:

$$
f^{\prime}(x) \approx \frac{f(x+\Delta x)-f(x)}{\Delta x}
$$

It is essential to remember that when finding a numerical solution for this model, the river is no longer treated as a continuous entity; rather, after a maximum value for $x$ is specified, it is broken up into a finite number of discrete locations, each separated by a distance of $\Delta x$. Similarly, after a maximum value for $t$ is specified, the dimension of time is also broken up into finitely many discrete points, each separated by an interval of $\Delta t$.

For computing convenience, let $C_{i, j}$ denote $C(x, t), C_{i, j+1}$ denote $C(x, t+\Delta t)$, and $C_{i+1, j}$ denote $C(x+\Delta x, t)$. The same notation holds for $N$. Translating (17) to our model's partial differential equations yields the following finite difference approximations:

$$
\begin{aligned}
& \frac{\partial C}{\partial t} \approx \frac{C_{i, j+1}-C_{i, j}}{\Delta t} \\
& \frac{\partial C}{\partial x} \approx \frac{C_{i+1, j}-C_{i, j}}{\Delta x} \\
& \frac{\partial N}{\partial t} \approx \frac{N_{i, j+1}-N_{i, j}}{\Delta t}
\end{aligned}
$$

These finite differences may be substituted into the original partial differential equations: 


$$
\begin{gathered}
\frac{C_{i, j+1}-C_{i, j}}{\Delta t}=-v \frac{C_{i, j}-C_{i-1, j}}{\Delta x}-\frac{\alpha C_{i, j}}{1+h N_{i, j}^{2}}+\left(\gamma N_{i, j}+\eta N_{i, j}^{2}\right) \\
\frac{N_{i, j+1}-N_{i, j}}{\Delta t}=\frac{\alpha C_{i, j}}{1+h N_{i, j}^{2}}-\left(\gamma N_{i, j}+\eta N_{i, j}^{2}\right)+r N_{i, j}\left(1-\frac{N_{i, j}}{K}\right)
\end{gathered}
$$

Using these two equations, it is possible to solve for both $C_{i, j+1}$ and $N_{i, j+1}$ as long as the state of the system is known at the $j^{\text {th }}$ point in time and the value $C_{i-1, j}$ is defined. Therefore, since the initial conditions $N(x, 0)$ and $C(x, 0)$ along with the boundary condition $C(0, t)$ are assumed to be known in the model, it is possible perform a simulation of the river that starts with the initial conditions and then iterates forward through time, updating the system using this finite difference method during each iteration. This process is known as the upstream finite difference scheme and is stable if $\frac{\Delta x}{\Delta t} \geq|v|$ [Morton/Mayers, 2005].

Based on this scheme, we wrote a program in $\mathrm{R}$ to simulate the behavior of this system under various circumstances. For the sake of simplicity, we assumed the initial condition of the system to be empty $(C(x, 0)=N(x, 0)=0)$ and the boundary condition $C(0, t)$ to be constant. Throughout the program, matrices are used to record values for $C$ and $N$ at each location and time. The kernel of the program consists of a nested loop that calculates values of $C$ and $N$ at all locations of the river - starting at $x=0$ using the boundary condition and then iterating forward to some specified maximum distance - and at all points in time - starting at $t=0$ using the initial conditions and then iterating forward to some specified maximum time. The following pseudocode describes the process:

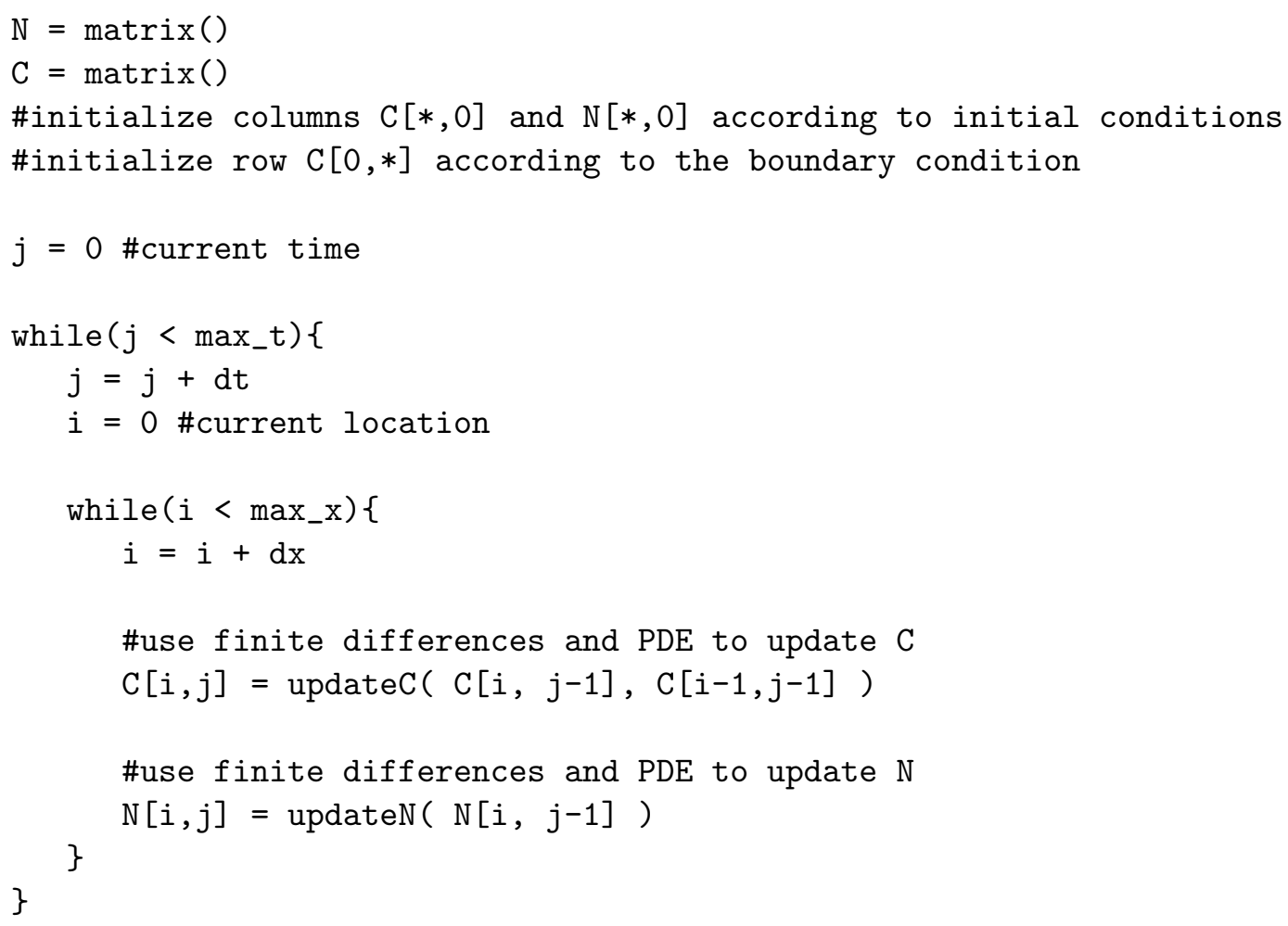




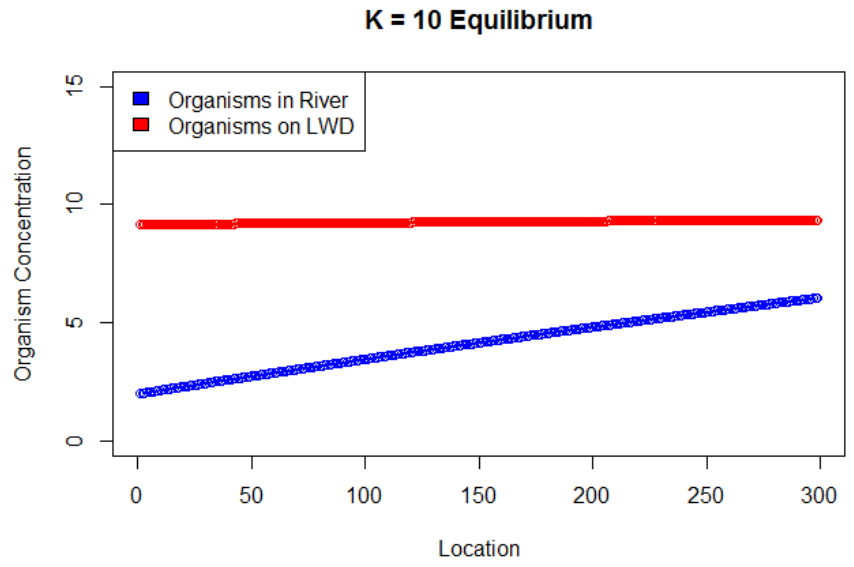

Figure 3: Simulated equilibrium using carrying capacity $K=10$

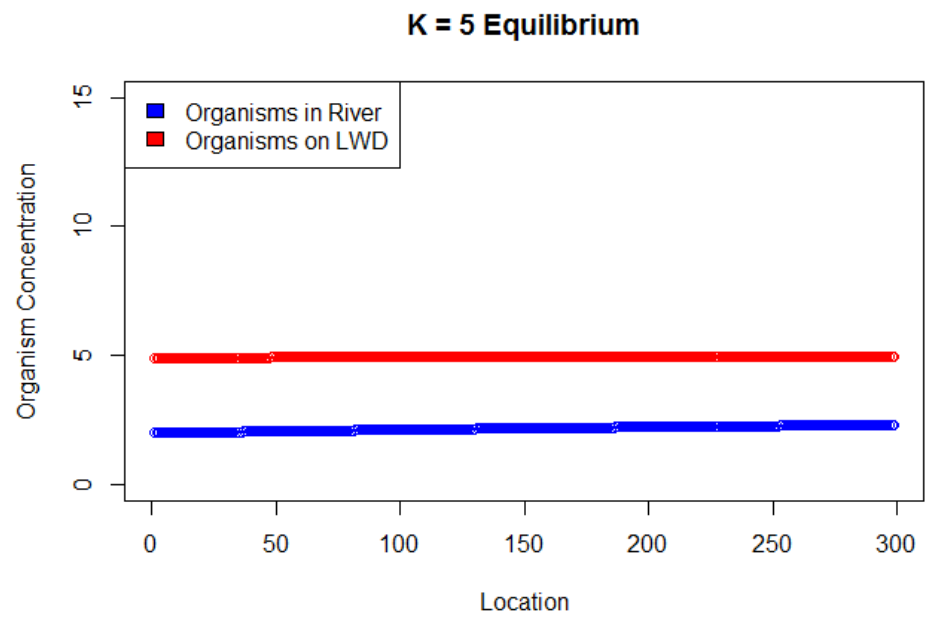

Figure 4: Simulated equilibrium using carrying capacity $K=5$

Figures 3 and 4 provide examples of the output of this program. Figure 3 was generated using the following combination of parameters:

$$
\alpha=0.1 v=1 ; h=0.1 ; \gamma=0.01 ; \eta=0.002 ; r=0.2 ; K=10
$$

The boundary condition constant was chosen to be 2 . Note that the carrying capacity $K=10$ is consistent with data from Roger Haro, et.al [Unpublished Data]. Figure 4 was generated using the same values for each parameter with the exception of $K$, which was reduced to 5 . This modification is analogous to decreasing the amount of large wood debris that is available for colonization in the river. It may be observed that reducing this parameter not only causes fewer organisms to be latched onto the large wood debris but also results in fewer organisms throughout the current of the river. 


\section{Competing Organisms Model}

Realistically, in the Upper Mississippi River, caddisfly larvae must compete with other invertebrates for space on large wood debris. One of these invertebrates is the invasive zebra mussel, which, like the caddisfly larva, latches on to structures such as large wood debris and filter-feeds on microorganisms in the river. The zebra mussel has proven to be a quite prolific species, and its presence in the UMR has the potential to significantly affect caddisfly larvae population.

In order to bring this competing organism into consideration, it is necessary to add two more partial differential equations to the system. The four dependent variables of the system are now $C_{1}, N_{1}, C_{2}, N_{2}$, where $C_{i}$ denotes the quantity of organism $i$ in the current of the river and $N_{i}$ denotes the quantity of organism $i$ on LWD. Each organism has its own parameters that govern the rate at which it latches onto LWD, reenters the current, and grows on the LWD. Also, these rates are affected by not only each organism's own population but also the population of the other organism:

$$
\begin{gathered}
l a_{1}=\frac{\alpha_{1} C_{1}}{1+h_{1} N_{1}^{2}+h_{2} N_{2}^{2}} \\
l a_{2}=\frac{\alpha_{2} C_{2}}{1+h_{1} N_{1}^{2}+h_{2} N_{2}^{2}} \\
l e_{1}=\gamma_{1} N_{1}+\eta_{1} N_{1}\left(N_{1}+N_{2}\right) \\
l e_{2}=\gamma_{2} N_{2}+\eta_{2} N_{2}\left(N_{1}+N_{2}\right) \\
f_{1}=r_{1} N_{1}\left(1-\frac{N_{1}+N_{2}}{K}\right) \\
f_{2}=r_{2} N_{2}\left(1-\frac{N_{1}+N_{2}}{K}\right)
\end{gathered}
$$

Note how, in the latching terms, both organism populations contribute to the overcrowding that reduces the proportion of organisms that will latch onto the large wood debris. Similarly, both populations contribute to the overcrowding portion of the leaving terms. Also, the carrying capacity $K$ is a shared parameter between the two growth rates such that as the sum of the two population totals approaches this value, neither organism will have space to grow on the LWD. The parameter $v$ is also assumed to be shared between the two populations, since it represents the velocity of the river. However, each population may have its own boundary condition and initial conditions.

The complete system of PDE's for the competition model is formed by combining the previous terms with two instances of the transport equation to yield: 


$$
\begin{gathered}
\frac{\partial C_{1}}{\partial t}=-v \frac{\partial C_{1}}{\partial x}-l a_{1}+l e_{1} \\
\frac{\partial C_{2}}{\partial t}=-v \frac{\partial C_{2}}{\partial x}-l a_{2}+l e_{2} \\
\frac{\partial N_{1}}{\partial t}=l a_{1}-l e_{1}+f_{1} \\
\frac{\partial N_{2}}{\partial t}=l a_{2}-l e_{2}+f_{2}
\end{gathered}
$$

The structure of the program that simulates this competition model is identical to that for the single-organism model. However, four matrices are now used to record values for each dependent variable and the upstream finite difference method is used four times to update these matrices at each location and time.

\section{Equilibrium with Similar Competing Organisms}

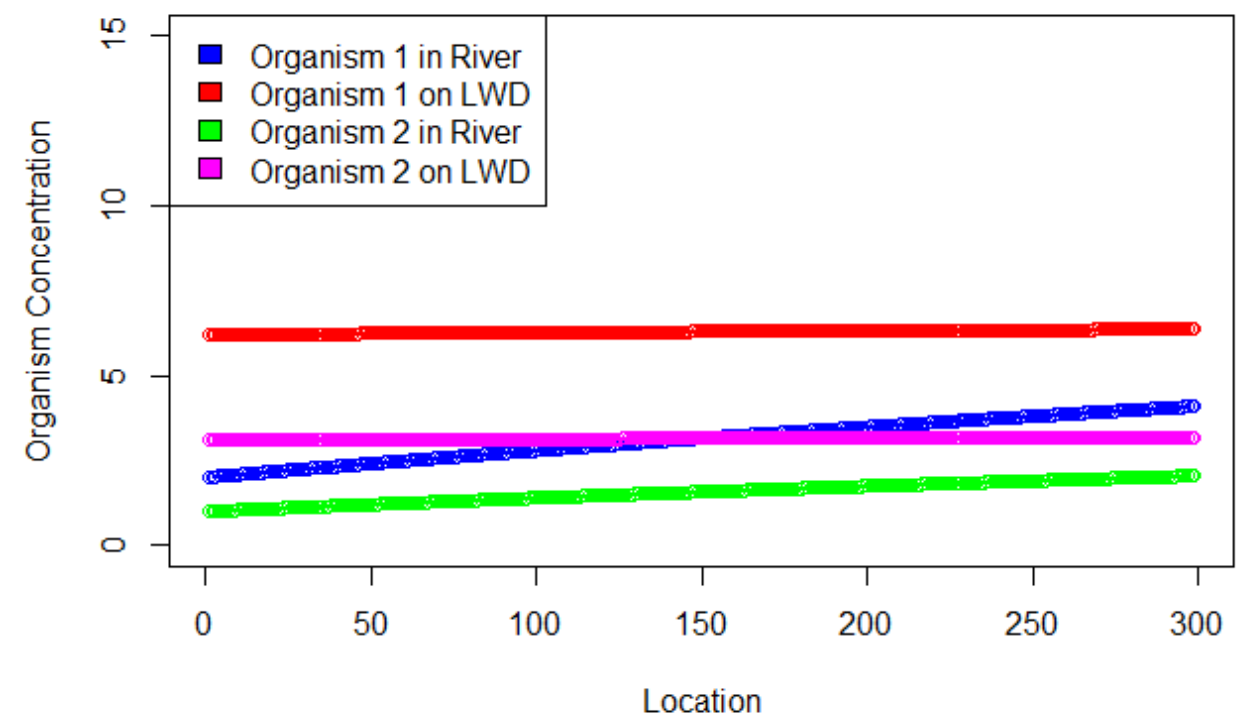

Figure 5: Equilibrium of competing organisms model with identical parameters

Figures 5 and 6 illustrate the simulated results of the competition model. In figure 5, two competing organisms share the parameters that were used to generate figure 3 :

$$
\alpha_{1}=\alpha_{2}=0.1 ; v=1 ; h_{1}=h_{2}=0.1 ; \gamma_{1}=\gamma_{2}=0.01 ; \eta_{1}=\eta_{2}=0.001 ; r_{1}=r_{2}=0.2 ; K=10
$$

However, the boundary condition constant for organism 1 remains 2 while the boundary condition for organism 2 is reduced to 1 . All initial conditions are 0 . Not surprisingly, the population of organism 1 roughly doubles that of organism 2 on the LWD at equilibrium. 


\section{Equilibrium with Differing Competing Organisms}

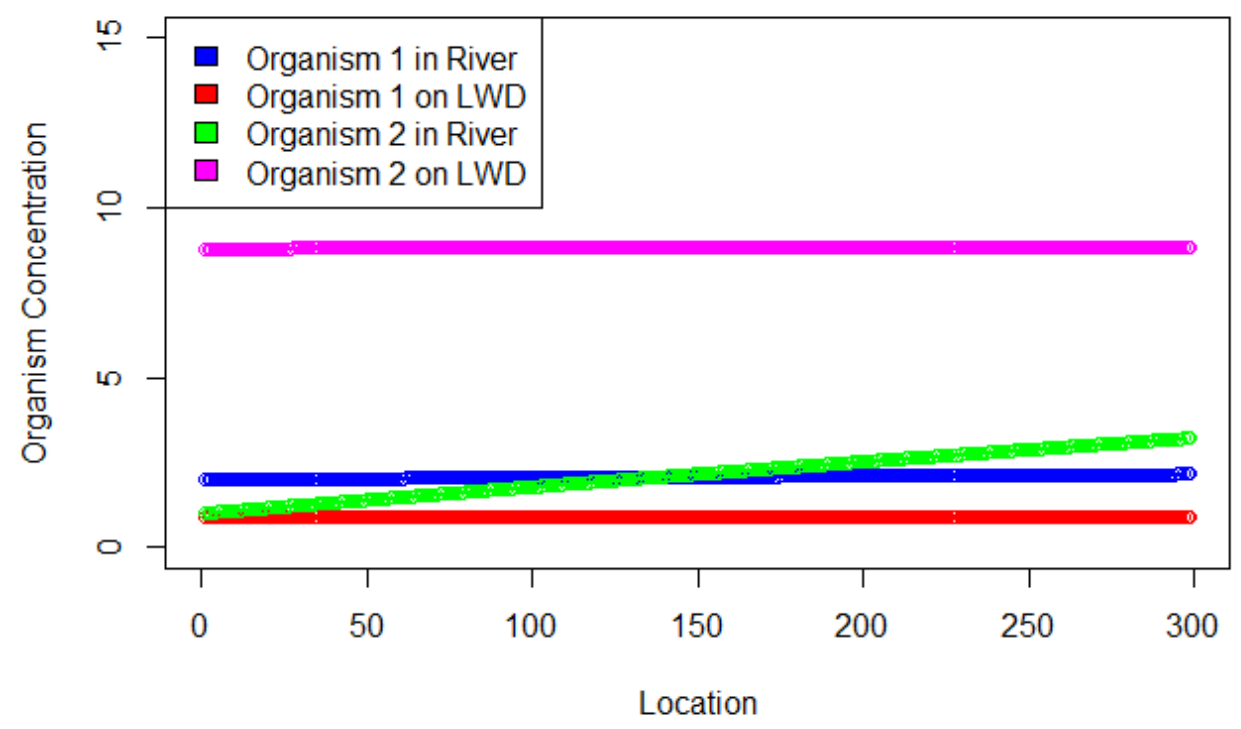

Figure 6: Equilibrium of competing organisms model with one organism dominant

On the other hand, figure 6 illustrates the effect of introducing a more prolific organism (such as the invasive zebra mussel) to the competitive model. Parameter changes were limited to increasing $h_{2}$ to 0.2 (implying that organism 2 contributes more to overcrowding); reducing $\gamma_{2}$ to 0.005 and $\eta_{2}$ to 0.0005 (implying that organism 2 is less likely to leave LWD); and increasing $r_{2}$ to 0.3 (implying that organism 2 grows faster once it is latched onto LWD). Boundary condition constants for organisms 1 and 2 remain at 2 and 1 respectively.

Although the boundary condition for organism 1 is double that for organism 2, the latter completely dominates the former on the LWD once equilibrium is reached. This simulation demonstrates the potential threat the zebra mussel poses to caddisfly larvae in the Upper Mississippi River. Although there is no direct interaction between the two organisms (one does not actually consume the other), an initially small population of zebra mussels can overtake the less prolific caddisfly larvae by increasing in numbers, reducing the amount of available resources in the river, and thereby preventing the larva population from attaining significant growth.

\section{Conclusion and Future Directions}

By using partial differential equations to describe how organisms are transported by the current of a river as well as how they interact with large wood debris scattered throughout the river, we have created a model that can predict and simulate the populations of lotic organisms such as the caddisfly larva and the zebra mussel in the Upper Mississippi River. As we have demonstrated, the model may be used to predict how certain parameter or resource changes may affect overall population growth. Also, the model may be used to capture the dynamics of competing organisms that rely on the same resource for population growth. 
Future directions for this model include determining which parameters, or sets of parameters, contribute the most toward a population's overall growth. For example - is it more beneficial for an organism to grow quickly while it is latched onto LWD or is it better for it to leave the LWD less frequently?

Additionally, the carrying capacity $K$ could be modified such that, rather than being a constant, it could be any function of $x$, allowing the quantity of LWD in the river to vary from location to location. In this way, different distributions of LWD could be compared in order to determine which ones allow for the greatest overall population growth.

Furthermore, a research question of interest is the inverse problem: given population data collected from wood debris in a river, can we accurately estimate realistic parameters for the model? One approach to this problem could be to use Latin hypercube sampling in order to discover a combination of parameters for our model that results in a simulated equilibrium that closely matches observed data.

Future directions such as these demonstrate that although understanding the precise behavior of lotic organism population remains a difficult task, mathematical models such as the one laid out in this paper can simplify the problem and serve as useful tools for future research.

\section{References}

[de Vries, 2006] Gerda de Vries, Thomas Hillen, Mark Lewis, Birgitt Schonfisch, Johannes Muller (2006) A Course in Mathematical Biology: Quantitative Modeling with Mathematical and Computational Methods Society for Industrial and Applied Mathematics

[Haro, 2014] Roger Haro (2014) Common Filter-feeding Caddisfly Larvae Breakdown Large Wood Debris in the Upper Mississippi River UW-L River Studies Center and Biology Dept.; Mathematics Dept.

[Morton/Mayers, 2005] K.W. Morton, D.F. Mayers. Numerical Solution of Partial Differential Equations: An Introduction. Cambridge University Press, 2005. University of Victoria

[Schloesser/Naleba/Mackie, 1996] Don W. Schloesser, Thomas F. Nalepa, and Gerald L. Mackie (1996) Zebra Mussel Infestation of Unionid Bivalves (Unionidae) in North America American Zoology 36(3), 300 - 310.

[Wiley, 1981] Michael J. Wiley (1981) Interacting Influences of Density and Preference on the Emigration Rates of Some Lotic Chironomid Larvae Ecological Society of America 62(2), 426 -438 .

[Zachmanoglou/Thoe, 1986] E.C. Zachmanoglou and Dale W. Thoe. Introduction to Partial Differential Equations with Applications. New York: Dover Publications, 1986. 